\title{
El uso de los yacimientos arqueológicos musealizados. Contextualización y caracterización de sus visitas en el ámbito de la Costa Brava
}

\section{The Use of Archaeological Sites Presented as Museums. Context and Characterization of Visits to Such Sites in the Area of the Costa Brava}

\author{
Gabriel AlCALDE \\ Facultad de Letras. Universidad de Girona \\ Plaça Ferrater Mora 1. 17071 Girona \\ gabriel.alcalde@udg.edu \\ Josep Burch \\ Facultad de Letras.Universidad de Girona \\ Josep.burch@udg.edu
}

Recibido: 30-05-2014

Aceptado: 30-07-2014

\begin{abstract}
Resumen
A principios del siglo XX el yacimiento arqueológico de Empúries abrió sus puertas a las visitas. Desde aquellos momentos hasta nuestros días numerosos yacimientos de la zona están preparados para ser visitados. Estas actuaciones, en muchas ocasiones, se encuentran relacionadas con su uso como elementos de atracción turística. En este trabajo se analizan las características del uso de estos yacimientos por parte de sus visitantes y se hace un especial hincapié en el interés de situar estas visitas en el contexto más general de las estancias turísticas o las salidas excursionistas.
\end{abstract}

Palabras Clave: Patrimonio arqueológico. Estudios de visitants. Costa Brava. Estudios turísticos.

\begin{abstract}
At the beginning of the twentieth century, the Empuries archaeological site opened its doors to visitors. Since then, numerous sites in the area have been made ready for visits. In many cases, the work that has been undertaken is related with the use of the sites as tourist attractions. In this paper, we analyse the characteristics of use of the archaeological sites by their visitors. In particular, we situate the visits in the more general context of tourist's holidays or day trips in the area.
\end{abstract}

KeY words: Archaeological Heritage. Visitor Studies. Costa Brava. Tourism Studies. 


\section{Introducción}

La Costa Brava se considera como un destino turístico maduro que ha llegado a un cierto estancamiento (Sardà, Mora y Ávila 2004). Para conseguir un cambio en el modelo que evitase que este estancamiento se convirtiera en declive, desde la década de los años 90 del pasado siglo se han desarrollado diversas estrategias, algunas de las cuales han impulsado la utilización de los recursos culturales en la oferta turística (Donaire, Fraguell y Mundet 1997). En los momentos actuales, la cultura y la identidad son considerados como productos de alto potencial para el desarrollo turístico de la zona y así queda reflejado, por ejemplo, en el Pla Estratègic de Turisme de les Comarques Gironines 2011-2015 (2011).

El patrimonio cultural, y de forma especial el patrimonio arqueológico, se ha incorporado de esta forma con fuerza a la oferta turística de la Costa Brava y la zona cuenta actualmente con diversos yacimientos arqueológicos museizados (Fig. 1). Este hecho queda reflejado, por ejemplo, en el folleto turístico Costa Brava Catalunya, editado en diversos idiomas el 2007 por la Generalitat de Catalunya, en el cual se concede una especial atención a los yacimientos arqueológicos de Empúries, Roses y Ullastret.

En este trabajo analizamos las características y las especificidades del uso de los yacimientos arqueológicos museizados, situados en la Costa Brava, en el marco del interés que despiertan entre la población local y de su papel en la oferta turística de la zona. El análisis se basa en los resultados obtenidos a partir de un estudio de visitantes de estos yacimientos, realizado en el marco del proyecto "La patrimonialización de la arqueología. Conceptualizaciones y usos actuales del patrimonio arqueológico"1 (Alcalde y Burch 2011).

\section{La museización de los yacimientos arqueológicos en la Costa Brava}

El interés por mostrar al público los resultados de las investigaciones arqueológicas realizadas en la zona fue muy temprano. En el año 1908, la Junta de Museos de Barcelona iniciaba las excavaciones arqueológicas de Empúries (Aquilué et al. 2008). En este mismo año empezaron a ser visitadas, y ya al año siguiente recibían grupos de visitantes procedentes de Barcelona. En 1917 se inauguró un pequeño museo de sitio, posterior-

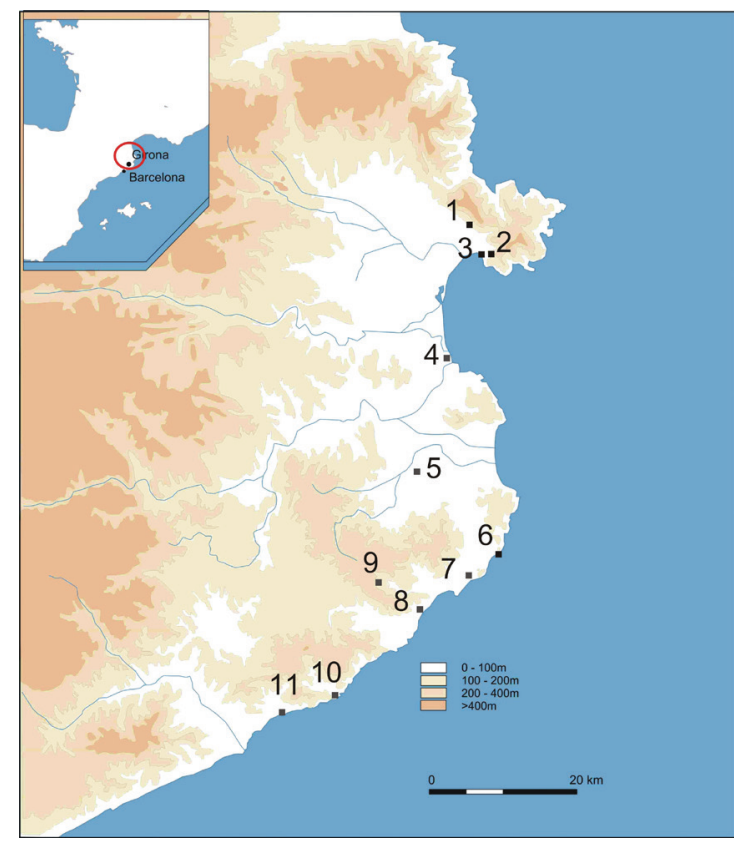

Fig. 1. Localización de los principales yacimientos arqueológicos musealizados de la Costa Brava (1: Can Issac, 2: Creu d'en Cobertella, 3: Roses, 4: Empúries, 5: Ullastret, 6: Sant Sebastià de la Guarda, 7: Castell, 8: Pla de Palol, 9: Cova d'en Daina, 10: Els Ametllers, 11: Puig Castellet).

mente se cercó el yacimiento, se empezaron a colocar paneles explicativos y a principios de los años treinta se cobraba una entrada para acceder al recinto arqueológico.

Actualmente, después de más de cien años de excavaciones y de recibir visitas, Empúries es el recinto arqueológico de mayor extensión y más visitado de la Costa Brava. La actual exposición permanente data de inicios de los años noventa, si bien fue complementada con una sala acondicionada expresamente para ello en el año 2008 mediante la exposición de la estatua original de Esculapio, el elemento más emblemático del museo.

Los restos arqueológicos que es posible visitar en Empúries son los correspondientes a una ciudad griega y otra romana (Fig. 2). De la griega se pueden observar, siguiendo el trazado original de las calles del núcleo antiguo, las numerosas estructuras arqueológicas, aunque, a excepción de la casa del Peristilo, de la zona conocida como Ágora y del espacio exterior de la muralla, no existe una adecuación específica para su visita. A lo largo del recorrido están instalados paneles explicativos que combinan textos en diversos idio- 


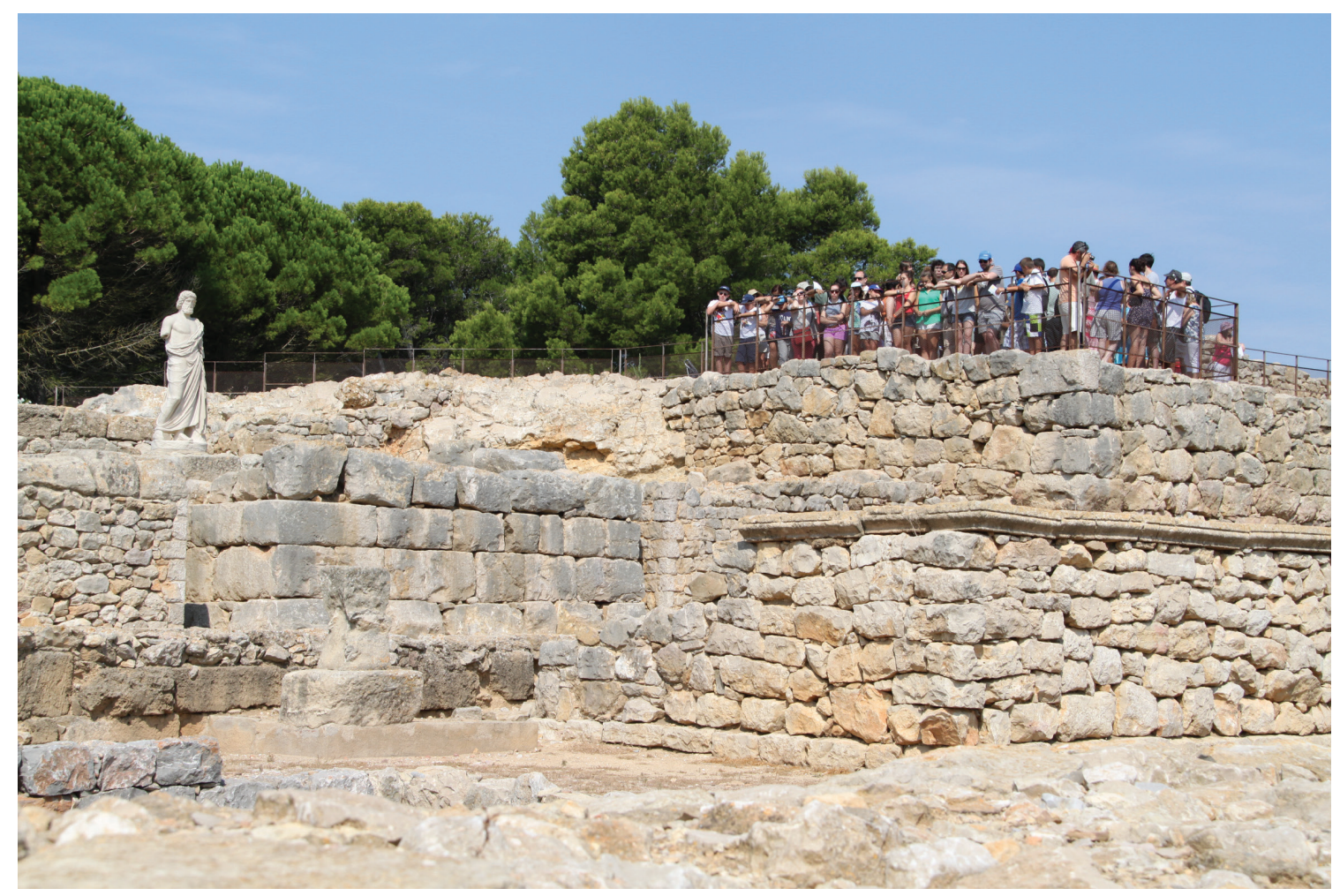

Fig. 2. Visitantes en la neápolis de Empúries (Foto autores).

mas con imágenes. Por lo que respecta a la ciudad romana, se pueden visitar dos casas, el foro de la ciudad, las termas públicas de la ínsula 30, el tramo meridional de las murallas y los restos del anfiteatro. De estos espacios, el más intensamente museizado es el del foro donde, entre otras actuaciones, se ha reconstruido una parte del $\mathrm{am}$ bulacrum (Aquilué y Tremoleda 2011).

Otro de los yacimientos arqueológicos ampliamente conocidos de la Costa Brava es el poblado ibérico de Ullastret, situado a unos $14 \mathrm{~km}$ del litoral. Las excavaciones arqueológicas se iniciaron en 1947 y se intensificaron a partir del año 1952. En 1961 se inauguraba un museo monográfico, sito en el mismo lugar, que fue ampliamente reformado entre los años 1995 y 1996 (Martín 1997). El yacimiento se integra actualmente en la "Ruta dels Ibers", que enlaza algunos de los principales asentamientos ibéricos de Cataluña. Entorno al 2005, el equipamiento se complementó con una ruta asociada a su entorno natural próximo (Baqué, Casas y Martín 2005). Los restos arqueológicos visibles no han sido profundamente museizados. En conjunto, la imagen proyectada es más cercana a la de un jardín arqueológico.
La Ciudadela de Roses es una fortificación militar de época moderna, en el interior de la cual se encuentran principalmente restos arqueológicos de la polis griega de Rhode y de la villa medieval de Roses (Díaz, Palou y Puig 1998), que cuenta desde el 2004 con un museo monográfico en su interior. El conjunto se halla situado en el núcleo urbano de Roses, ubicado en el mismo litoral. Los restos de época antigua, que fueron museizados a inicios de siglo XXI, son los correspondientes a un barrio de la polis griega y los relativos a una factoría de salazón de pescados de época romana. En ambos casos, los trabajos de museización consistieron en la pavimentación de los niveles de circulación, la consolidación de estructuras y su iluminación con la finalidad de facilitar las visitas nocturnas. En relación a la villa medieval la museización consistió en la consolidación de las estructuras y su inclusión en el itinerario de visita del conjunto.

Además de estos tres sitios arqueológicos, diversos yacimientos arqueológicos museizados de menor entidad se distribuyen a lo largo de la Costa Brava. La Creu d'en Cobertella (Roses) es un sepulcro de corredor que fue completamente ex- 
cavado y restaurado en 1957. Actualmente, está protegido por una valla dispuesta a su alrededor, está dotado de paneles explicativos e integrado en una ruta megalítica creada por el ayuntamiento de Roses y en uno de los itinerarios del Parque Natural del Cap de Creus (Alcalde et al. 2013). La Cova d'en Daina (Santa Cristina d'Aro) es una sepulcro megalítico de galería cubierta excavado en 1957 y restaurado en los años 1979 y 1986, que actualmente es de libre acceso y está dotado con paneles explicativos escritos en diversos idiomas. En el municipio de Palau-savardera se encuentra el poblado neolítico de Can Isaac, excavado entre 1987 y 1994 y posteriormente museizado entre los años 2001 y 2003, con la restauración de los restos de diversas cabañas, la reconstitución de una de ellas y la colocación de paneles informativos y de una valla perimetral (Tarrús y Carreras 2004).

Sant Sebastià de la Guarda (Palafrugell) es un poblado ibérico excavado entre 1998 y 2008 y museizado en el año 2009; de acceso libre, presenta una museización basada en la adecuación de los niveles correspondientes a su última fase arqueológica y en la creación de un itinerario interior. Puig Castellet es otro de los poblados ibéricos museizados en la Costa Brava; situado en Lloret de Mar, su acceso está restringido a los horarios de visita; la museización de este yacimiento consistió en la consolidación de las estructuras del poblado y en la creación de un itinerario, complementado con paneles informativos sobre el poblado y sobre la fauna y la flora del entorno (Fuertes et al. 2002). El tercer poblado ibérico museizado de la zona es el de Castell (Palamós), que es de acceso libre y cuenta con una museización basada en la consolidación de diversos muros, la recuperación de los niveles de circulación ibéricos más modernos y la colocación de diferentes paneles explicativos (Aquilué y Tremoleda 2011).

La museización de restos arqueológicos de época romana, Empúries al margen, viene representada por las villas de Pla de Palol, en Platja d'Aro, y Els Ametllers, en Tossa de Mar. La primera fue museizada en el año 2002 mediante la consolidación de las estructuras, la pavimentación de los ámbitos, la colocación de paneles y la creación de un itinerario, que forman parte de una plaza pública integrada al entorno urbano de la población (Burch y Figueras 2002). La villa romana de Els Ametllers, excavada en diversos períodos desde 1914 hasta 2004, fue museizada en este último año; su actual presentación ha dado como resultado la recuperación de diversos niveles de circulación, la consolidación de estructuras y la creación de un itinerario interior (Palahí y Nolla 2010; Burch, Figueras y Sureda 2005).

En conjunto, la zona de la Costa Brava, cuenta con once yacimientos museizados (además de numerosos megalitos señalizados y algún elemento muy puntual como los restos de una prensa de vino romana hallada en Llafranc, en Palafrugell). De estos once yacimientos, la entrada es de pago en cuatro y tres disponen de museos monográficos. A excepción de reconstrucciones parciales y muy puntuales en algunos yacimientos, la museización ha consistido básicamente en la consolidación de sus estructuras originales, la pavimentación de los niveles de circulación y la colocación de paneles explicativos. Empúries, Ullastret y Castell están gestionados por el Museo de Arqueología de Catalunya, mientras que el resto de yacimientos han sido museizados por iniciativas de los correspondientes ayuntamientos, a cargo de los cuales se encuentra también su gestión.

\section{El volumen de visitas}

Con el objetivo de conocer la evolución que se ha producido en el uso de los yacimientos arqueológicos, se dispone para la zona de la Costa Brava de datos sobre el volumen de visitas de aquellos yacimientos para los que es preciso obtener un tique para acceder a ellos. De forma concreta, se dispone de información sobre visitas a Empúries, Ullastret y Roses. En este trabajo nos centraremos en analizar la evolución de las visitas en los últimos cincuenta años, si bien las informaciones de que se dispone para cada yacimiento corresponden a períodos distintos, de acuerdo con la historia de la museización de cada uno de ellos. Para efectuar el análisis de Empúries se ha trabajado con datos sobre afluencia de visitas desde 1960, para Ullastret desde 1983 y para Roses desde 2004.

Las cifras sobre afluencia de visitas son difícilmente comparables de forma precisa puesto que las metodologías utilizadas en los distintos momentos de recuento y en los diferentes yacimientos no son perfectamente homologables. Sin embargo, las cifras de que se dispone permiten determinar las tendencias que se producen en su evolución. El gráfico correspondiente a las visitas a Empúries muestra una primera fase que se sitúa entre 1960 (momento de inicio de nuestro 
análisis) hasta mediados de la segunda mitad de la década de los años 70 (1977, 1978), y que se caracteriza por una tendencia al aumento de visitas; una segunda fase, que transcurre desde mediados de la segunda mitad de la década de los 70 hasta los primeros años del siglo XXI (2005), y que muestra una estabilización del volumen de visitas, si bien con diversas fluctuaciones; y una tercera fase, entre 2005 y el momento actual, que determina una tendencia a la disminución del volumen de visitas.

El gráfico relativo a la evolución del volumen de visitas de Ullastret, muestra un primer momento caracterizado por un incremento del volumen de visitas, que se sitúa entre 1983 y 1986, seguido por un amplio período de una cierta estabilización, si bien con numerosas fluctuaciones, hasta inicios del siglo xx (2002), momento a partir del cual se inicia una tendencia a la disminución que se prolonga hasta el momento actual.
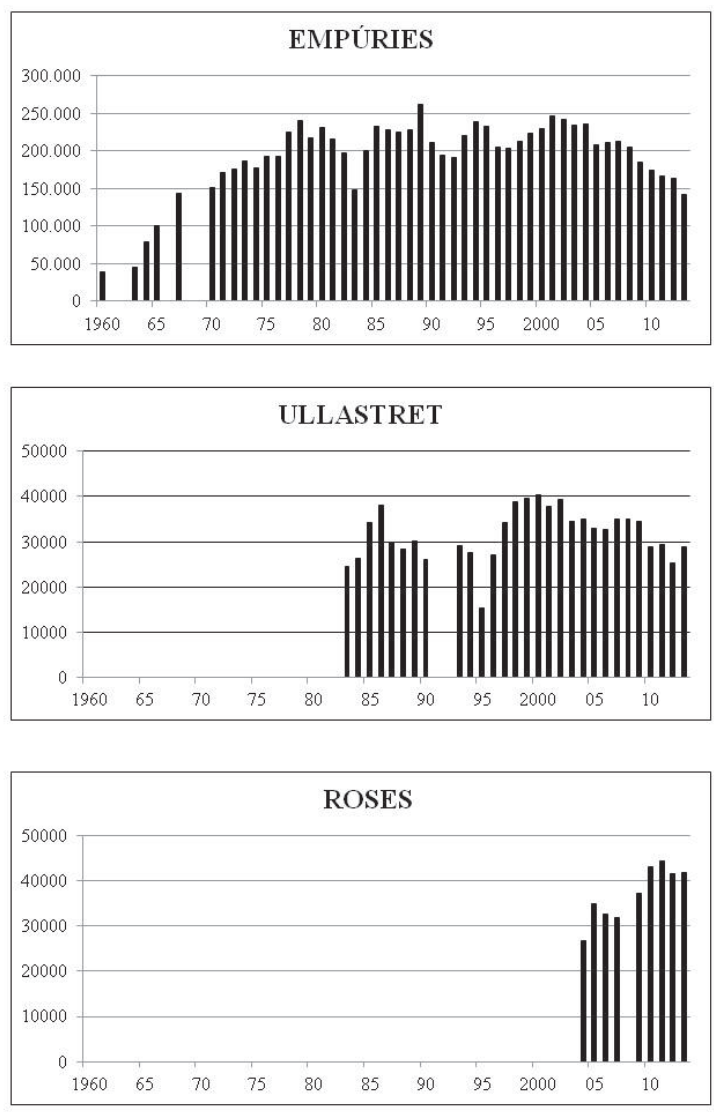

Fig. 3. Diagramas con la repartición del volumen de visitas a los yacimientos arqueológicos de Empúries, Ullastret i Roses (Fuente: Generalitat de Catalunya).
Para Roses las posibilidades de análisis del volumen de visitas se diferencian de los anteriores yacimientos analizados a causa de que su museización es muy reciente. En un primer momento el volumen de visitas de este yacimiento experimenta un período de incremento desde su apertura hasta el inicio de la década de 2010 (2011), momento en el cual se observa una cierta estabilización que será preciso confirmar en los próximos años (Fig. 3).

Además de las tendencias que muestran las evoluciones del número de visitas, se puede también concretar el volumen y el impacto que adquieren las visitas a estos tres yacimientos museizados. Si se toma la media de los últimos diez años (2004-2013), para evitar efectos sobre un año concreto, se puede decir que Empúries recibe anualmente unas 190.000 visitas, Ullastret unas 31.000 y Roses unas 37.000 .

\section{Características de las visitas}

Para obtener un conocimiento preciso sobre las características de los visitantes y de las visitas a los yacimientos arqueológicos analizados se dispone de las informaciones obtenidas en un programa de encuestas realizadas a los visitantes de los yacimientos de Empúries, Roses, Castell y Sant Sebastià de la Guarda (con un total de 1.946 encuestas realizadas en los meses de julio, agosto y septiembre de 2010).

Los datos obtenidos en este estudio permiten disponer de informaciones diversas. En relación con las edades de los visitantes de los cuatro yacimientos arqueológicos las franjas más frecuentes son las que se sitúan en los 31-35 y 4145 años, decreciendo la presencia de visitantes de edades inferiores y superiores a estas franjas. Mayoritariamente, se trata de personas con formación universitaria y que en una proporción muy importante tienen su residencia en el extranjero o en el resto de Catalunya (fuera de la localidad donde se encuentra el yacimiento). La visita en familia o en pareja es muy frecuente y para muchos de los visitantes se trata de la primera vez que visitan el yacimiento. Una proporción importante de visitantes están alojados en un municipio distinto al que se encuentra el yacimiento, y muchos de ellos se alojan en casa de amigos o familiares o en segundas residencias. En general se observa que no se trata de un conjunto de personas especialmente practicantes de actividades arqueológicas, si bien muchos 
A: EDAD DE LOS VISITANTES

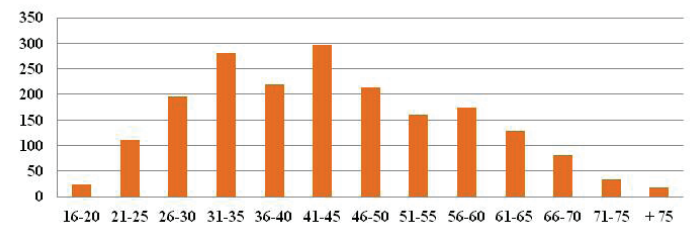

B: NIVEL DE ESTUDIOS

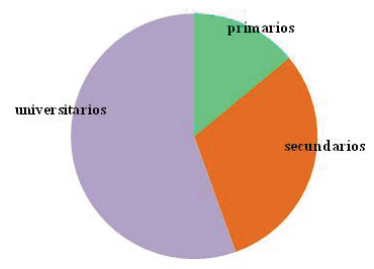

D: ALOJAMIENTO

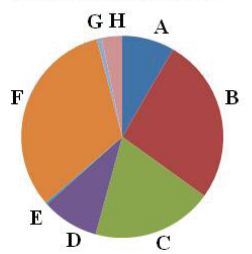

C: RESIDENCIA HABITUAL

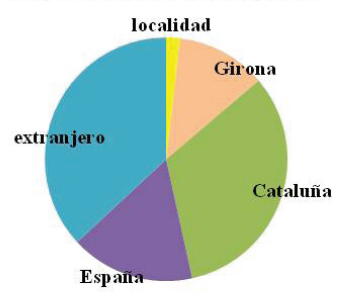

E: PRIMERA VISITA

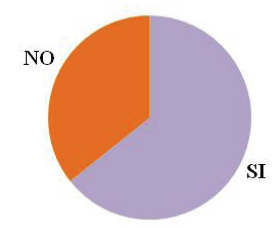

Fig. 4. A: edad de los visitantes, B: nivel de estudios, C: residencia habitual (localidad del yacimiento, provincia de Girona sin la localidad, Catalunya sin la provincia de Girona, España sin Cataluña, extranjero), D: tipo de alojamiento utilizado en el día en que se realiza la visita al yacimiento (A: residencia habitual; B: segunda residencia; C: hotel, hostal, pensión; D: camping, autocaravana; E: casa de colonias, albergue; F: casa de amigos o familiares; G: casa rural; H: apartamento alquilado para vacaciones) y E: Primera visita al yacimiento, en los cuatro yacimientos analizados (Castell, Roses, Empúries y Sant Sebastià).

de ellos conocían con anterioridad la existencia del yacimiento que visitan (Fig. 4).

Con el objetivo de obtener una visión conjunta de las diferentes características de los visitantes de los cuatro yacimientos analizados y al mismo tiempo poder determinar si existen unas características comunes a todos ellos, se ha realizado un análisis de correspondencias múltiples ${ }^{2}$ para cada yacimiento y sobre el conjunto de los cuatro yacimientos.

Este análisis, aplicado a los resultados obtenidos en las encuestas de Castell, muestra la presencia de tres grupos diferenciados en relación a la

dinámica de la visita. El grupo 1 (202 individuos) se caracteriza por estar formado por personas que realizan por primera vez la visita al yacimiento $(94,55 \%)$ y por personas que han obtenido la información sobre el yacimiento a través de canales diversos $(56,44 \%)$. El segundo grupo (126 individuos) está constituido por personas que ya han realizado la visita al yacimiento con anterioridad $(98,41 \%)$ y por personas que han obtenido la información sobre el yacimiento a partir de sus anteriores visitas $(87,30 \%)$. Finalmente, el tercer grupo ( 82 individuos) está formado principalmente por personas que realizan la visita en el marco de una salida de un único día $(98,78 \%$ ) y por personas que se alojan en el día de la visita en su residencia habitual (58,54 \%) (Fig. 5).

Entre los visitantes encuestados en Roses se distinguen tres grupos de visitantes con características diferenciadas. El grupo 1 (21 individuos) está caracterizado por estar formado por personas que realizan una salida de un solo día $(95,24 \%)$ y por personas que se alojan el día de la visita en su residencia habitual $(66,67 \%)$. El grupo 2 (53 individuos) está formado por personas que conocen el yacimiento de anteriores visitas $(88,62 \%)$ y por personas que han obtenido la información sobre el yacimiento en anteriores visitas $(83,02$ $\%)$. El tercer grupo, que cuenta con un peso muy superior a los dos anteriores grupos (229 individuos), está formado por personas que realizan su primera visita al yacimiento $(97,38 \%$ ) y por personas que han obtenido la información sobre este a través de oficinas de turismo y de elementos de información turística (44,10 \%) (Fig. 5).

Para el yacimiento de Empúries se han establecido tres grupos de visitantes. Un primer grupo, con un peso medio (228 individuos), está formado por personas que han visitado con anterioridad el yacimiento $(76,04 \%)$ y por personas que han obtenido la información sobre el sitio en sus anteriores visitas $(83,68 \%)$. Un grupo 2, de menores proporciones (106 individuos) caracterizado por estar formado por personas que residen en la provincia de Girona $(80,19 \%)$ y por personas que hacen una salida de un único día $(95,28 \%$ ) y que se alojan el día de la salida en su residencia $(90,58$ $\%$ ). El tercer grupo es el mejor representado (448 individuos) y está formado por personas que realizan su primera visita a Empúries $(93,75 \%$ ) y por personas que han obtenido la información sobre el yacimiento en oficinas de turismo o a través de productos turísticos $(59,60 \%)$ y por personas que en el momento de realizar la visita residen en casa de amigos o familiares (65,85 \%) (Fig. 5). 


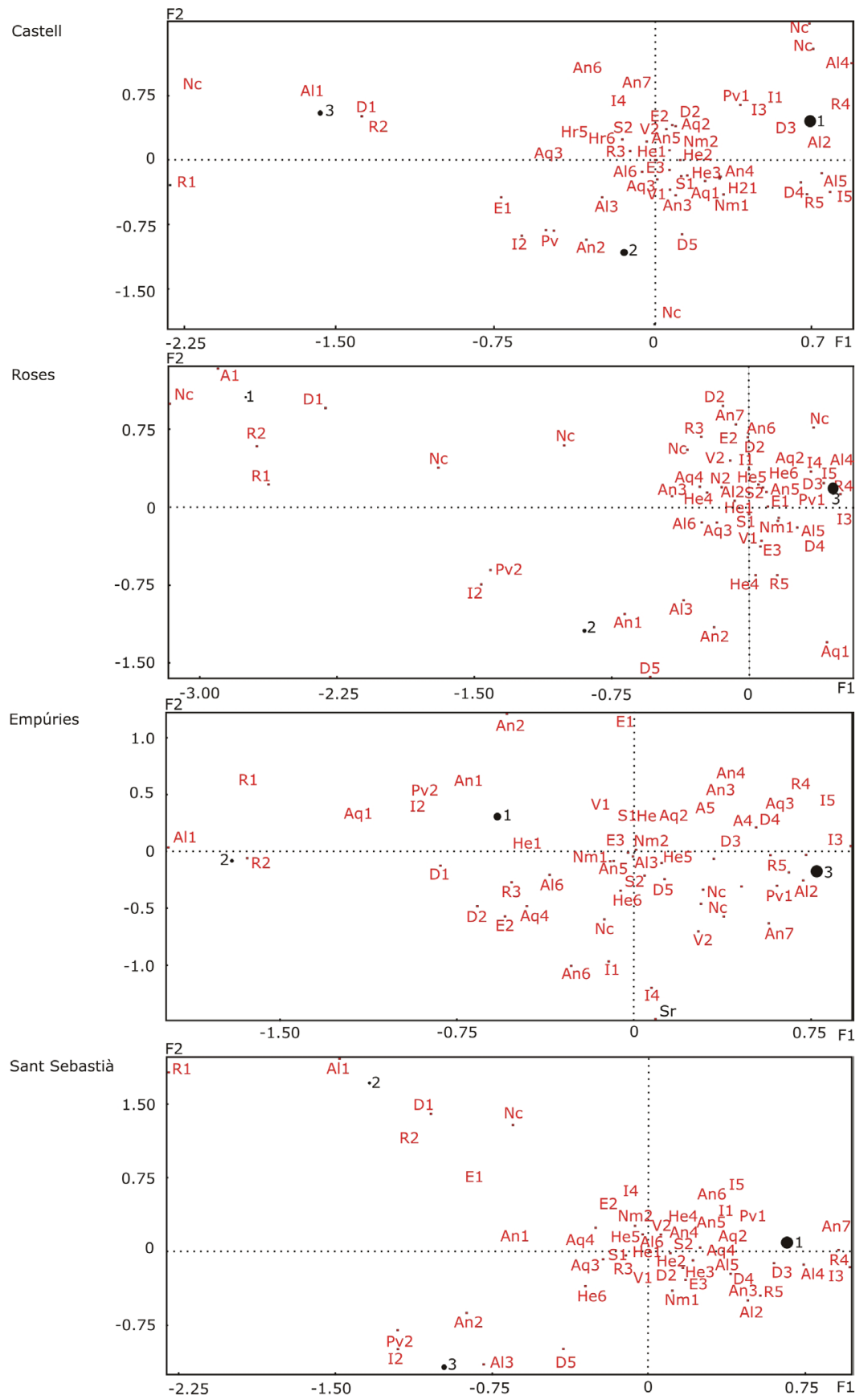

Fig. 5. Análisis de correspondencias múltiples de los datos de las encuestas de visitantes a cada uno de los cuatro yacimientos arqueológicos analizados (Castell, Roses, Empúries y Sant Sebastià) (Factores 1 y 2).

En el análisis de las visitas a Sant Sebastià se han diferenciado cuatro grupos. El grupo 1, que es el mejor representado (221 individuos), está formado por personas que realizan por primera vez la visita al yacimiento $(98,77 \%)$ y por personas que han obtenido información sobre el yacimiento a partir de canales diversos $(71,31 \%)$.
Dos otros grupos tienen poca representación, el grupo 2 (71 individuos) con personas que residen en la provincia de Girona $(53,52 \%)$ y por personas que realizan una salida de un único día $(85,92$ $\%$ ) y el grupo 4 (90 individuos) formado por personas que ya habían visitado el yacimiento en anteriores ocasiones $(92,55 \%)$ y por personas 


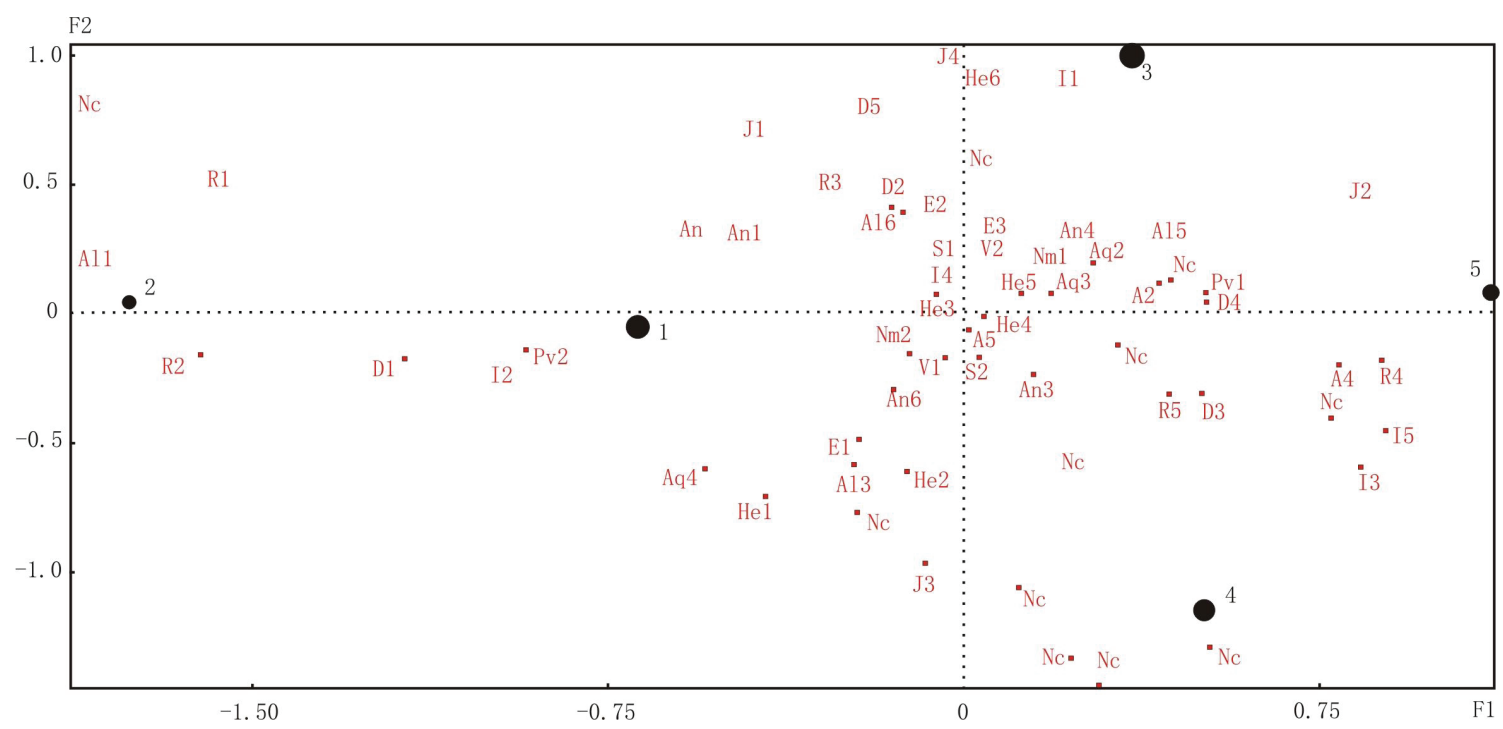

Fig. 6. Análisis de correspondencias múltiples del conjunto de los datos de las encuestas de visitantes a Castell, Roses, Empúries y Sant Sebastià (Factores 1 y 2).

que han obtenido información sobre el yacimiento de anteriores ocasiones $(89,36 \%)$. El grupo 3 con un volumen muy reducido de efectivos ( 8 individuos) se caracteriza por estar formado por personas residentes en el municipio donde se encuentra el yacimiento $(100,00 \%)$ y por personas que en el día de la visita se alojan en su residencia habitual (87,5 \%) (Fig. 5).

En los cuatro casos analizados se observa como el parámetro que más influye en la formación de los diversos grupos es el conocimiento previo del yacimiento y la información aportada por las oficinas de turismo y por los diferentes elementos de promoción turística (folletos, guías, carteles, información en hoteles...). Así, los grupos con visitantes que ya conocen el yacimiento son más importantes en Roses, mientras que en Empúries, Castell y Sant Sebastià son más numerosos los grupos constituidos por personas que no conocían previamente el yacimiento.

Para obtener un análisis global de perfiles de visitantes de los cuatro yacimientos se ha realizado también un análisis del conjunto de los datos obtenidos, utilizando el método de clasificación jerárquica.

A partir de las asociaciones de la dimensión 1 , pueden observarse dinámicas diferenciales entre los yacimientos de Castell y Empúries, por un lado, y el yacimiento de Roses por otro. Mientras que a partir de la segunda dimensión los yacimientos Castell y Sant Sebastià presentan una misma dinámica, en contraposición al yacimiento de Empúries.

La clasificación jerárquica evidencia la presencia de cinco grupos diferentes que corresponderían a dinámicas de visita diferentes:

- Del grupo 1 (473 individuos) la mayoría $(93,87 \%)$ de sus componentes corresponde a visitantes que cuentan con información previa sobre el yacimiento, habiéndolo visitado la mayoría más de una vez, y su visita no está especialmente vinculada al efecto de los canales de información turística.

- El grupo 2 (242 individuos) lo constituyen los visitantes de la zona, residentes la mayoría en la misma provincia $(64,05 \%)$ y que no pasan ninguna noche fuera de casa $(99,12 \%)$.

- El grupo 3 (478 individuos) lo constituyen los visitantes que obtienen información a partir de canales diversos $(69,67 \%)$ y que realizan por primera vez la visita al yacimiento $(95,40 \%)$. El $50 \%$ son visitantes de Sant Sebastià, mientras que el 38,91\% son visitantes de Castell, y se encuentran muy pocos visitantes de Empúries (4,18 \%).

- El 97,41 de individuos del grupo 4 (425 individuos) son visitantes del yacimiento de Empúries y este grupo está formado principalmente por visitantes extranjeros $(72,24$ $\%$ ) y que han conocido la existencia del yacimiento a partir de los principales canales de información turística $(57,18 \%)$. 
- El grupo 5 (327 individuos) corresponde a visitantes españoles que residen en el resto de España (fuera de Cataluña) (58,72\%), que realizan la primera visita al yacimiento. La mayoría de las personas de este grupo $(66,67$ \%) son visitantes del yacimiento de Roses.

A partir de estos datos podemos concretar que la visita a los cuatro yacimientos analizados está muy condicionada por la información que se dispone sobre los mismos. Los yacimientos de Sant Sebastià y Castell reciben, por ejemplo, sobre todo visitas de personas residentes en la zona mientras que Empúries atrae a visitantes extranjeros, probablemente debido a su mayor difusión entre el sector turístico. Se evidencia asimismo una dinámica diferencial en la visita a los yacimientos según el lugar de residencia de los visitantes (Girona, Cataluña, España, extranjero). Estas diferencias pueden atribuirse a las mismas estrategias de difusión que se implementan desde los diferentes yacimientos: Empúries sigue una estrategia más global e intenta atraer a visitantes interesados por la problemática histórica o cultural del mismo yacimiento, logrando atraer de esta forma a visitantes extranjeros y consiguiendo también que las personas lo visiten en más de una vez; Roses, en cambio, presenta unos resultados más relacionados con el turismo local, igual que Sant Sebastià y Castell (Fig. 6).

\section{Contextualización de las visitas}

Muchos de los estudios de visitantes realizados en elementos patrimoniales se centran exclusivamente en los propios visitantes y en la visita al bien patrimonial. Este tipo de análisis puede resultar parcial e insuficiente para la compresión de los visitantes y sus visitas puesto que, en muchos casos, éstas se inscriben en un contexto más general de salidas turísticas o excursionistas en las cuales el bien patrimonial puede no constituir ni el principal ni el único motivo de la salida. Es por este motivo que, al mismo tiempo que consideramos de gran interés el conocimiento concreto del uso de los elementos patrimoniales, pensamos que es imprescindible contextualizar las visitas en el marco más general de las salidas en las cuales estas se incluyen (Alcalde 2013).

En este sentido, el estudio de visitantes realizado constata que en el día en el que se realiza la visita a un yacimiento arqueológico, esta no constituye, en la mayoría de los casos, la ocupación central y más importante del día. La posibilidad de ser la principal actividad del día solo se constata en casos muy puntuales, que se pueden considerar una excepción, y que se relacionan especialmente con la visita a Empúries. En estos casos, la visita ocupa una parte importante de la jornada y se suele complementar, en los meses de verano, con una estancia complementaria en la playa.

El análisis realizado identifica otro grupo (cuantitativamente poco numeroso y únicamente representado en Empúries y Roses) para el que la visita al yacimiento arqueológico si bien ocupa una parte importante del día, entre dos y cuatro horas, ya no es la actividad principal. Si los visitantes que se incluyen en este grupo se encuentran de vacaciones en la zona su visita al yacimiento se combina con otras actividades que ocupan una proporción más significativa de la jornada. La ocupación más frecuente es la de estar en la playa, o bien la de visitar algunos sitios, como pueblos o, de manera más puntual, algunos otros yacimientos arqueológicos, monumentos o museos, normalmente próximos al yacimiento visitado.

Para otro grupo, la visita al yacimiento arqueológico es una ocupación secundaria en términos temporales; la combinación de actividades y la casuística de los visitantes de este grupo es muy diversa y es mucho más variada que en los grupos anteriores. No obstante, la asociación con la playa en los meses de verano continúa siendo destacable. Así pues, una parte considerable de visitantes pasa el día en la playa y efectúa una visita puntual y muy acotada en el tiempo al yacimiento arqueológico (este hecho es especialmente constatable en el relación a Castell). En otras ocasiones, la playa y la visita al yacimiento se combina con la visita a otros lugares de manera individualizada o enlazados en forma de ruta. Estos lugares se hallan cercanos unos a otros aunque, a diferencia del grupo anterior, se pueden localizar algo más alejados, aunque siempre en el marco de las comarcas gerundenses.

Un último grupo se asocia a visitas breves, y se concretan en los yacimientos menos extensos como Castell o Sant Sebastià de la Guarda. En estos casos, las combinaciones de actividades en las cuales se inscriben las visitas a los yacimientos arqueológicos son las mismas que en los ejemplos anteriores: principalmente la playa y la visita a otros lugares, generalmente cercanos. 
Una singularidad de este grupo, muy encuadrada en Castell, es que una parte importante de estas visitas se enmarcan en excursiones a pie que resiguen los caminos de ronda del litoral. En este caso, las visitas al yacimiento son simples paradas en el recorrido.

\section{Conclusiones}

Como hemos visto, la presentación y adecuación de los yacimientos arqueológicos para su visita cuenta con una amplia tradición en la Costa Brava. Desde la apertura al público de Empúries, en los primeros años del siglo xx hasta nuestros días, han sido numerosos los yacimientos que se han museizado. Cronológicamente, la mayoría de estas museizaciones se sitúan principalmente a principios del siglo XXI y son proyectadas por la posibilidad de su uso como elemento de promoción turística (Alcalde y Burch 2006). En este marco, algunos ayuntamientos de la zona han apostado por la valorización de determinados yacimientos arqueológicos y han participado en los trabajos de investigación de estos yacimientos, los cuales han culminado con su museización.

Siguiendo la estela ampuritana, los yacimientos arqueológicos están en estos momentos contribuyendo a transmitir una determinada imagen de la Costa Brava, asociada directamente (las ciudades griegas de Emporion y Rhode, la ciudad romana de Emporiae y diferentes villas romanas) o indirectamente (poblados ibéricos que coexistieron con Empúries) a la época clásica. Al mismo tiempo dotan de una cierta singularidad a la oferta turística de la zona. Sin em- bargo, está siendo difícil que la participación en la imagen de la zona que se quiere transmitir se traduzca en una utilización notable de estos yacimientos tanto por parte de las comunidades locales como por parte de los turistas que en un volumen importante se desplazan a la zona.

Para el conocimiento de las características del uso de los yacimientos arqueológicos por parte de la sociedad se evidencia que no resulta suficiente un análisis de la propia visita, sino que se hace imprescindible que este análisis se amplíe al conjunto de las actividades que, en el marco de las salidas excursionistas o turísticas, desarrollan sus visitantes.

Entre quienes visitan los yacimientos analizados se encuentra una notable proporción de personas que realizan la visita en el marco de una salida turística y que obtienen información sobre los yacimientos a través de canales de información turística. Al mismo tiempo, también muchos de sus visitantes son personas que residen en zonas próximas al yacimiento y para ellas la visita al yacimiento se enmarca en una salida de un único día. En ambos casos, la visita a los yacimientos arqueológicos difícilmente es el principal motivo del desplazamiento a la zona específica donde estos se hallan, pero sí que constituyen un elemento que complementa y enriquece el desplazamiento.

Probablemente, el reto futuro para el incremento del uso del patrimonio arqueológico de la Costa Brava, será conseguir encontrar fórmulas que aumenten su capacidad de atracción, despertando un mayor interés al obtenido hasta ahora y así logrando su inclusión en los programas de las personas que deciden visitar o establecerse durante algunos días en la zona.

\section{Notas}

1. Ministerio de Ciencia e Innovación, HAR2008-00132/HIST.

2.Para el análisis de correspondencias múltiples se han utilizado 13 variables: nombre del yacimiento, hora de entrada, sexo, año de nacimiento, nivel de estudios, con quién realiza la visita, dónde reside, pasa o no la noche en el municipio, cuántas noches pasa fuera de su domicilio, dónde se aloja, cómo ha obtenido la información sobre la existencia del yacimiento, si es la primera visita, si visita habitualmente yacimientos o museos arqueológicos.

\section{REFERENCIAS Bibliográficas}

Alcalde, G. (2013): Analysis of Tourist Visits to the Historic Centre of Santa Pau as Part of Visit to the Garrotxa Volcanic Zone (Girona, Spain). International Journal of Humanities and Social Science, 3-7: 74-80. 
Alcalde, G.; Burch, J. (2006): L'ús del Patrimoni històric com a recurs turístic a la Costa Brava. El turisme i el mar. El patrimoni marítim i els nous turismes, Ajuntament de Palamós, Universitat de Girona, L'Estrop, Palamós: 55-61.

Alcalde, G.; Burch, J. (ed.) (2011): Conceptualitzacions i usos actuals del patrimoni arqueològic al nordest de la península ibérica. Institut Catalá de Recerca en Patrimoni Cultural, Girona.

Alcalde, G.; Burch, J.; Martí, E.; Rojas, A. (2013): Monumentos Megalíticos y Paisaje: Patrimonialización y uso del dolmen de la Creu d'en Cobertella (Roses, Cataluña). VI Congreso Internacional de Musealización de yacimientos y patrimonio. Arqueología, Patrimonio y Paisajes Históricos para el siglo XXI, Consorcio de Toledo, Toledo: 215-219.

Aquilué, X.; Castanyer, P.; Monturiol, J.; Oliveras, C.; Santos, M.; Tremoleda, J. (2008): 100 anys d'excavacions arqueologiques a Empúries (1908-2008). Museu d'Arqueologia de Catalunya-Ajuntament de l'Escala, Girona.

AquiluÉ, X.; Tremoleda, J. (2011): La museïtzació actual dels jaciments arqueològics. La patrimonialització de l'arqueologia. Conceptualitzacions i usos actuals del patrimoni arqueològic al nord-est de la península ibérica, Institut Català de Recerca en Patrimoni Cultural, Girona: 47-57.

Baqué, C.; CASAs, S.; Martín, A. (2005): El yacimiento ibérico de Ullastret: de la excavación a la contextualización. III Congreso Internacional sobre Musealización de yacimientos arqueológicos. De la excavación al público. Procesos de decisión y creación de nuevos recursos, Ayuntamiento de Zaragoza, Zaragoza: 229-234.

Burch, J.; Figueras, M. (2002): La consolidació i adequació de la vil·la romana de Pla de Palol (Platja d'Aro, Baix Empordà). Sisenes Jornades d'Arqueologia de les comarques de Girona, Museu d'Arqueologia de Catalunya, Girona: 465-468.

Burch, J.; Figueras, M.; Rojas, A.; Vivo, J. (2012): Musealization to the Iberian Oppidum at Sant Sebastià de la Guarda (Palafrugell, Baix Empordà) and its Public Use. The Arcaheological Musealization. Multidisciplinary Intervention in Archaeological Sites for the Conservation, Comunication and Cultur, Umberto Allemandi \& C., Turín: 63-69.

Burch, J.; Figueras, M.; Sureda, R. (2005): Patrimonio Cultural, turismo y desarrollo local. El ejemplo de Tossa de Mar. III Congreso Internacional sobre Musealización de yacimientos arqueológicos. De la excavación al público. Procesos de decisión y creación de nuevos recursos, Ayuntamiento de Zaragoza, Zaragoza: 203-207.

Díaz, C.; Palou, H.; Puig, A.M. (1998): La Ciutadella de Roses. Diputació de Girona y Caixa de Girona, Girona.

Donaire, J.A.; Fraguell, R.M.; Mundet, Ll. (1997): La Costa Brava ante los nuevos retos del turismo. Estudios Turísticos, 133: 77-96.

Fuertes, M.; Pons, E.; Teixidor, E.; Vidal, J.R. (2002): Campanyes de condicionament i consolidació al poblat ibèric de Puig Castellet (Lloret de Mar, La Selva). Sisenes Jornades d'Arqueologia de les comarques de Girona, Museu d'Arqueologia de Catalunya, Girona: 455-456.

Martín, A. (1997): Guies del museu d'Arqueologia de Catalunya. Museu d'Arqueologia de Catalunya, Girona.

Pla Estratègic de Turisme de Les Ccomarques Gironines 2011-2015 (2011): Patronat de Turisme Costa Brava Girona, Girona.

SARDÁ, R.; Mora, J.; Ávila, C. (2004): La gestión integrada de zonas costeras como instrumento dinamizador de la industria turística en la Costa Brava. XIII Simposio Internacional de Turismo y Ocio ESADE - Fira de Barcelona, ESADE - Fira de Barcelona, Barcelona: 1-28.

TARRÚs, J.; CARRERAS, E. (2004): La restauració i adequació per a la visita del poblat neolític de Ca n'Isach. Campanyes 2002-2003 (Palau-Saverdera, Alt Empordà). Setenes Jornades d'Arqueologia de les comarques de Girona, Museu d'Arqueologia de Catalunya, Girona: 71-77. 